\title{
Survey of the use of Point of Care Testing Devices in a Tertiary Hospital in Nigeria
}

Dr. Kenneth Ogar Inaku ${ }^{1 *}$ (MBBCh, FMCPath), Dr. Asuquo Bassey Ene ${ }^{1}$ (MBBS, FMCPath), Dr. Idongesit Kokoabasi Isong $^{2}$ (BSc, PhD), Dr. Okokon Ita Ita ${ }^{3}$ (MBBCh, FMCPath), Dr. Marcus Aniekan Inyama ${ }^{4}$ (MBBCh, FMCPath)

${ }^{1}$ Department of Chemical Pathology, Faculty of Medicine, University of Calabar, Calabar, Nigeria

${ }^{2}$ Department of Medical Laboratory Sciences, Faculty of Allied Medical Sciences, University of Calabar, Calabar, Nigeria

${ }^{3}$ Department of Medical Microbiology and Parasitology, Faculty of Medicine, University of Calabar, Calabar, Nigeria

${ }^{4}$ Department of Haematology and Blood Transfusion, Faculty of Medicine, University of Calabar, Calabar, Nigeria

DOI: $10.36348 /$ SJPM.2019.v04i10.003

| Received: 29.09.2019 | Accepted: 08.10.2019 | Published: 21.10.2019

*Corresponding author: Kenneth Ogar Inaku

\section{Abstract}

Laboratory testing plays a critical role in healthcare delivery with test results used for disease screening, diagnosis, treatment progress and prognosis. Some tests are performed outside the laboratory by non-laboratory trained individuals using Point of Care Testing (POCT) devices. We aimed to evaluate the knowledge, factors that may affect results generated, quality control practices and the importance of POCT regulation on the overall testing process. This is a prospective study which 70 POCT devices operators / users gave their consent at the University of Calabar Teaching Hospital. Pretested questionnaires were used as the tool for evaluation. The respondents were 70 in number including medical doctors (51.4\%) and Nurses (45.7\%). The most commonly used POCT devices included glucose meters (91.4\%), urine testing strips (65.7\%) and haemoglobin meters (25.7\%). Other devices in use were bilirubin meters (5.7\%) and cholesterol meters $(1.4 \%)$. About half $(56 \%)$ of respondents understood the test reaction principles of their devices. Accident and Emergency department had the highest use (37.1\%). Quality control practices were undertaken by $24.3 \%$ of respondents. Majority (65\%) of operators used their devices for monitoring the progression of illness. Opinion on the regulation of use of POCT was supported by most respondents. Periodically validating and calibrating POCT devices within the hospital by the central laboratory will go a long way to ensure uniform testing and improve result quality.

Keywords: Point-of-care-testing, Glucose meter, Urine testing strip, Quality control, Calibration, Screening.

Copyright @ 2019: This is an open-access article distributed under the terms of the Creative Commons Attribution license which permits unrestricted use, distribution, and reproduction in any medium for non-commercial use (NonCommercial, or CC-BY-NC) provided the original author and sources are credited.

\section{INTRODUCTION}

Laboratory testing plays a critical role in healthcare. Test results contribute to screening, diagnosis, and prognosis of diseases including the monitoring of the progress of treatment. It has been said that laboratory test results influence up to $70 \%$ of medical decisions [1]. Testing of body fluids and secretions can be done within or outside a laboratory environment. Tests performed outside the laboratory (also known as point-of-care-testing [POCT]) has been called various other names including near-patient testing, bedside testing, physician office testing, off site testing, and alternative site testing etc. This contrast with the historical pattern where testing was mostly confined to the medical laboratory requiring collection and transportation of specimens away from the point of care and then waiting hours or days to receive the results. During the waiting time, care must continue without the desired information [2].
An Irish group who recently completed a survey of POCT in Irish hospitals defined POCT as "a quality-assured pathology service" using analytical devices (including test kits and analyzers such as blood gas and critical care analyzers, meters for glucose; urinalysis and other metabolites) provided near the patient rather than in the traditional environment of the clinical laboratory [3]. Advances In technology has made testing simpler, thereby contributing to this shift in tests location. Many tests can now be performed using compact or handheld devices by personnel with limited experience and training [4]. Hence, POCT can be performed generally in diverse locations such as at home (self-testing), pharmacy shop, ambulance, nursing home, or aged care centre. Other locations include physician office laboratory, critical care facility in major hospitals, hospital ward or clinic, workplace drug screening and a lot more other locations. 
Some major advantage of POCT includes shorter turnaround time, ease of use, lower cost and more patient involvement in their management. Some devices have memory for easy result reference. However, in spite of numerous evident advantages, use of POCT has some drawbacks. Because the test is easily available, there is a tendency to increased inappropriate testing and performance by inexperienced, non-laboratory trained staff. Analytical method, reference intervals and results may differ from those of the laboratory thus making comparison difficult. Quality control may be inadequate due to poor training and the results may be lost due to poor documentation. There is also the concern about patient safety while performing POCT and there are documented reports of adverse outcomes [5].

Results from POCT can be used to adjust medication dosages, as in the case of blood glucose, bilirubin monitoring; or patients on anticoagulation therapy. The implication of an erroneous result may prove to be dangerous or even fatal. In the United States of America (USA) for example, POCT is classified by Clinical Laboratory Improvement Amendments (CLIA) as waived tests with negligible risk for erroneous results. There is therefore no requirement for personnel qualification and training [6, 7]. However, facilities performing only waived tests are required by law to obtain a Certificate of Waiver (CW), pay biennial certificate fees, and follow manufacturers test instructions. A survey of such sites in 2001 revealed some gaps in good laboratory practices including failure to follow manufacturer's instructions or failure to identify incorrect results and performing unauthorised testing [8]. Although the impacts of such errors or nonconformances on patient care were not evaluated, the report identified the need for education and training of testing personnel especially at sites where Certificate of Waiver has been issued.

The use of POCT is growing rapidly in Nigeria. They are used by clinical laboratories within and outside the hospital, pharmacy shops, and even by patients themselves or their relatives. There is no legal requirement regulating the operation of POCT nor is there specification of minimum qualification or training. In hospitals where these test are performed, there are no policies guiding their operations. There is evidence that the range of tests that can be carried out using POCT is increasing by the day. We therefore surveyed the use of POCT devices in our environment. We ask the range of available POCT devices, the minimum qualification and training of the operators and how much they are trying to follow the manufacturer's instructions to ensure a good outcome. The results from this survey will be used to improve on the quality of results generated from POCT devices in our environment.

\section{MATERIALS AND METHODS}

Setting: This is a prospective study involving a survey of persons who routinely use the different POCT devices in the University of Calabar Teaching Hospital. Informed consent was obtained from all participants after explaining the purpose of the survey to them. However, for the purpose of this survey they were requested to fill the questionnaire.

\section{Survey Instrument}

The study involves the use of pretested questionnaire developed by the researchers. The questionnaire domains were designed based on level of education, experience, existing literature and input from practice leaders. A pretest of the questionnaire was conducted among a small group of doctors in the Laboratory Medicine departments $(n=20)$ and the outcome was used to refine the instrument in terms of clarity and interpretability of the questions. The questionnaire was designed to evaluate: (1) knowledge of operations and maintenance of POCT devices,(2) attitudes and practices about quality control testing, and (3)possible effect of regulatory laws on use of POCT devices in Nigeria.

\section{Respondents Characteristics}

We asked about the level of education of respondents, profession of the operator of the POCT device, designation and years of practice if medical or paramedical.

\section{Knowledge of operation of the POCT device}

We inquired about the type of device(s) operated, frequency of use, place of use and department/section of the hospital. We also asked about the source(s) of training received on the operation of the device and the interpretation of the results generated. What steps are taken in the event of an abnormal result and any precaution taken to ensure that the instrument is kept in good condition?

\section{Quality Control Practices}

Respondents were asked about knowledge of any pretesting patient preparation requirements, use of quality control material before testing the unknown, and if they ever visited the central laboratory in the hospital to compare their test results periodically. We also inquired about their opinion on periodically testing a quality control sample before the unknown.

\section{Regulation of the use of POCT devices}

Respondents were asked if they think the clinical laboratory should have a role to play in the recommendation and validation of a POCT device before it is put to use especially in a hospital setting. We also sought their opinion about the effects of regulation by Government on the quality of results generated. 


\section{Study Population}

All persons who routinely perform point of care testing within the hospital facility or privately at home were eligible for recruitment into the study.

Inclusion Criteria: All who routinely perform tests using POCT device and gave consent to participate in the survey.

\section{Exclusion Criteria}

Those who do not handle POCT devices regularly and all those who did not give written consent to take part in the survey

\section{Ethical Consideration}

Ethical clearance was obtained for this evaluation from the Cross River State Health Research Ethics Committee.

\section{DATA COLLECTION}

The data was collected by administering the pretested questionnaire to persons who handle POCT devices in the hospital. The researchers or their trained assistants administered the questionnaire to consenting participants. The questionnaire was filled out by the respondents if they were literate or they were aided by the researchers or their assistants to complete it.

\section{STATISTICAL ANALYSIS}

Data obtained was entered into Microsoft excel (2007) and exported to Statistical Package for
Social Sciences (SPSS) version 20.0 for analysis. Descriptive analyses were carried out to quantify responses and presented as frequencies and percentages. A bivariate analysis was done to determine associations between knowledge of the operation of POCT devices and socio-demographic characteristics (gender, level of education), profession (medical, paramedical and nonmedical) among study participants. They were added simultaneously with a separate model for each outcome. A $p$-value of $\leq 0.05$ was accepted as statistically significant.

\section{RESULTS}

\section{General Characteristics of Respondents}

The respondents were 70 in number including $36(51.4 \%)$ medical doctors, $32(45.7 \%)$ nurses, one (1.4\%) medical laboratory scientist and a (1.4\%) respondent whose profession was not indicated. Majority of them 39(55.7\%) had acquired tertiary education while $31(44.3 \%)$ were undergoing their postgraduate training.

The most commonly used POCT devices among the respondents included glucose meters (91.4\%), urine testing strips $(65.7 \%)$ and haemoglobin meters $(25.7 \%)$. Other devices in use were bilirubin meters $(5.7 \%)$ and cholesterol meters $(1.4 \%)$.More than half $(56 \%)$ of respondents knew the test reaction principles of the instrument they were using as shown in Figure 1.

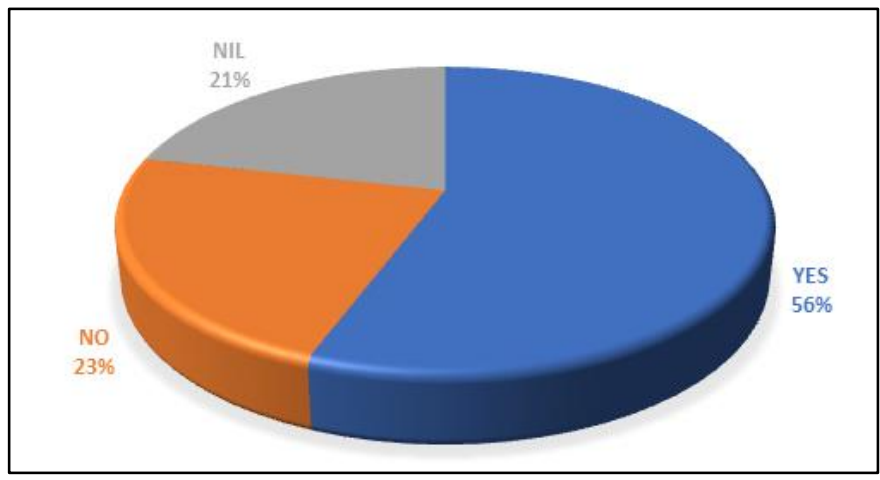

Fig-1: Percentage distribution of the knowledge of the principles of operation of POCT devices

Most POCT devices were used within hospitals $(92.9 \%)$, medical laboratories $(5.7 \%)$ and pharmacy shops $(4.2 \%)$. Some are also used in private homes (24.3\%). POCT devices used within the hospital facility are deployed most often in the Accidents and Emergency (A\&E) departments (37.1\%), Side laboratories (30.0\%), and clinics (20\%). They were also used in the wards $(8.6 \%)$ and less often in the central laboratory $(1.4 \%)$. The number of tests carried out using POCT devices ranged from as low as 3 to as high as 180 tests per month with an average of about 16 tests performed monthly. The section of the hospital with the highest use of POCT devices is the A\&E department where as much as 150 to 180 testing can be performed each month. Forty-two (60\%) operators of POCT devices received training from colleagues, while $28.6 \%$ studied the product leaflet provided by the manufacturer. Others received instruction by their product vendor $(14.3 \%)$, friends with similar devices $(10 \%)$ and a few others from unspecified sources of training (4.3\%).

Table 1 contains data on quality control practices and steps taken to ensure that the instrument is functioning optimally. Among those who admitted to the use of quality control materials in their testing, $29.4 \%$ do so weekly, $35.3 \%$ do so monthly while $17.7 \%$ include control once in 6 months. Twelve respondents 
(70.6\%) obtained control materials from medical laboratories (58\%), instrument vendors $(25 \%)$ and pharmacy shops (16\%). On the use of POCT devices, 34 $(48.6 \%)$ respondents use their devices to screen for diseases, 32(45.7\%) make diagnoses and 46(65.7\%) to monitor progress of an illness. The inclusion of control materials was considered to be unnecessary and a waste of strips by $4 \%$ of respondents while majority $(61 \%)$ considered it very beneficial. Thirty-seven percent were unsure about the value of control testing.

Table-1: Instrument Validation and Quality Control Practices by Respondents

\begin{tabular}{|c|c|c|c|c|}
\hline Variable & Frequency & Percentage & $\chi^{2}$ & $P$ - value \\
\hline \multicolumn{5}{|c|}{ Inclusion of Control Testing } \\
\hline Yes & 17 & 24.3 & & \\
\hline No & 35 & 50 & & \\
\hline Nil & 18 & 25.7 & & \\
\hline Total & 70 & 100 & 8.771 & 0.012 \\
\hline Total & 59 & 100 & 70.407 & $0.000 *$ \\
\hline \multicolumn{5}{|c|}{ Special Precaution to Keep The Instrument Safe } \\
\hline Yes & 62 & 88.6 & & \\
\hline No & 1 & 1.4 & & \\
\hline Nil & 7 & 10 & & \\
\hline Total & 70 & 100 & 96.886 & $0.000^{*}$ \\
\hline \multicolumn{5}{|c|}{ Results Comparison with Central Laboratory } \\
\hline Yes & 33 & 57.9 & & \\
\hline No & 23 & 40.4 & & \\
\hline Nil & 1 & 1.7 & & \\
\hline Total & 57 & 100 & 28.211 & $0.000^{*}$ \\
\hline
\end{tabular}

Table 2 contain respondents' view about the effect of regulation of the use of POCT devices in the hospital setting. Half of respondents agree that there is need for POCT devices to be sent to the clinical laboratory for validation of manufacturer's claims before they are put into routine use. They agree that Hospital Management should set up a committee to oversee the operation of POCT devices within the hospital. Some of the functions of the committee as identified by respondents should include: recommendations for the brand of POCT device(s) to be used $(62.5 \%)$; validation of manufacturer's claims about the device $(56.3 \%)$; periodic re-calibration of the device $(68.8 \%)$; comparing device methodology to routine laboratory method (65.3\%); and training/education of POCT devices handlers (79.2\%). Twenty-two $(31.4 \%)$ respondents were silent about the possible functions of the committee.

Table-2: Respondents opinion about Regulation of the use of POCT devices in the Hospital

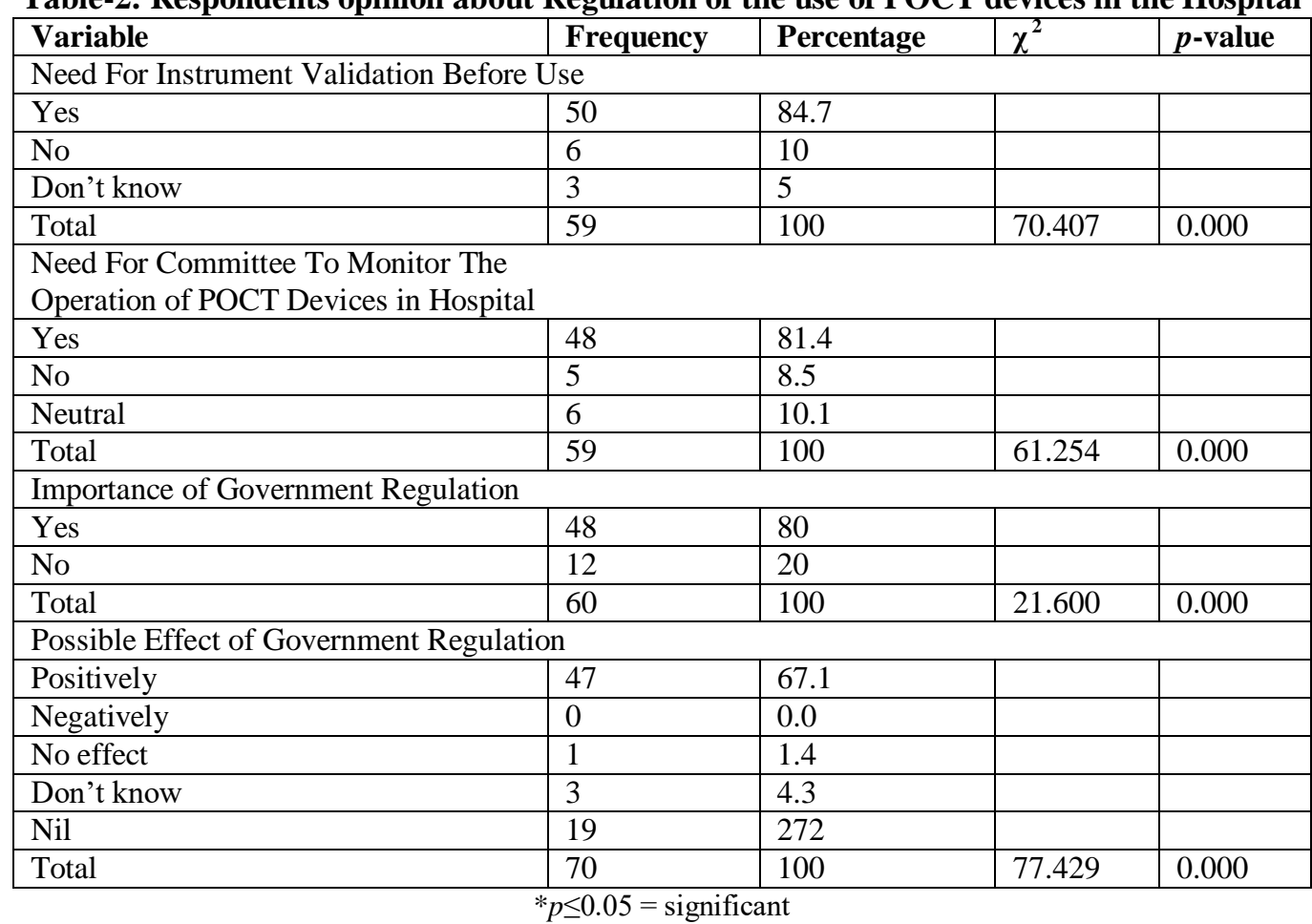




\section{DISCUSSION}

Extra-laboratory testing of patient's sample or point of care testing is common practice in our environment. We found that majority of POCT takes place within the hospital setting. Operators of POCT devices were also found to be medical or paramedical professionals. This is probably the first study that has looked at the use of POCT devices in our environment and perhaps in Nigeria. The most common POCT devices in use include glucose meters, urine testing strips, haemoglobin meters, bilirubin meters and cholesterol meters. There are other devices which were not captured in this study used within the hospital including POCT devices for measuring creatinine, urea, glycated haemoglobin, troponin, etc.

Since the operators of the POCT devices in this survey were medical professionals with some understanding of pathophysiology of the conditions they are dealing with they have a reasonable understanding of the results generated. The implications are that they will provide good interpretation and put the results to proper use. However, the reference values provided by the device manufacturer may not always apply to our populations due to unique socio-economic factors that may affect test results [9]. This may require that users of POCT devices check with the central laboratory to confirm if the values recommended by the manufacturers should be adopted rather than the locally generated reference intervals. To decide on this, the central laboratory will need to confirm the method used by the POCT device. If the method is same as the laboratory method in use, the already accepted reference intervals can be adopted. In the event of different methodology, there may be need to carry out comparison of method experiment to establish a relationship between the routine method in use in the central laboratory and the POCT device methodology. By using such comparative method, it is possible to demonstrate the accuracy and the quantitative performance of the device. This is demonstrated by plotting allowable total error (ATE) and limits of erroneous results (LER) [10].

In the USA the use of POCT devices are to some extent regulated by law. The Food and Drug Administration (CLIA '88) guidelines clearly states thus: "The examinations and procedures [that may be performed by a laboratory with a Certificate of Waiver] are laboratory examinations and procedures that have been approved by the Food and Drug Administration for home use or that, as determined by the Secretary, are simple laboratory examinations and procedures that have an insignificant risk of an erroneous result, including those that -- (A) employ methodologies that are so simple and accurate as to render the likelihood of erroneous results by the user negligible, or (B) the Secretary has determined pose no unreasonable risk of harm to the patient if performed incorrectly[10]." This statement implies that possible harm to a patient when the result from a POCT is false is negligible. It can be assumed that POCT devices may not be used to make diagnosis on medical conditions without recourse to confirmation using approved laboratory methods. In this survey, about half of respondents admitted using their devices to make some diagnoses of medical conditions of patients who may be presenting even for the first time. This may be misleading and may pose a great danger to patients as repeat tests or paired results provide confirmation of diagnosis. The use of POCT devices in a hospital setting as an alternative to the central laboratory may therefore constitute misuse and/or abuse of POCT. There is need to restrict its use to emergency conditions to aid initial management such as in A\&E department or Intensive Care Units (ICU) where urgent decisions are needed to prevent further harm.

Clinical Laboratory Improvement Amendments (CLIA) has no specific requirement for qualification of personnel allowed to operate POCT devices. This is because the tests are simple to perform with no particular need for routine quality control and proficiency testing [10]. But this may be fast changing. The American Association of Clinical Chemistry (AACC) is currently running an online program on Point of Care Testing Professional Certification [11]. It is designed to train personnel who perform diagnostic tests outside the central laboratory and will equip certified personnel in near patient testing, device selection and validation, quality management, operator training and more. In our study, almost all our respondents have acquired some training in medicine or medicine-related discipline. They had also learnt to operate their devices through various means including learning from colleagues and product leaflets etc. The importance of a formal training cannot be overemphasised. Many of them admitted to practicing quality control testing and periodic comparison of results generated with that of the central hospital laboratory. Although these claims were not verified, the Chemical Pathology Laboratory in our hospital has a record of a few POCT devices sent for calibration. The importance of this can be shown in a reported case of a 45 year old man with history of tricuspid valve replacement on warfarin therapy, digitalis and beta blockers. He has been monitoring his international normalized ratio (INR) in the past 6 months using CoaguChek S® (Roche Diagnostics, Indianapolis, Indiana). He developed complications of thrombosis despite 12 INR consecutive results ranging from 2.6 to 3.9 with his therapeutic range of 3.0-4.0. A comparison of laboratory INR result (2.9) with POC INR result (4.3) revealed that patient was actually getting inadequate anticoagulation therapy due to misleading results from his POCT device. He was treated and the POCT device was replaced with a more stable one. The lesson learnt is that POC INR devices require periodic 
checks to ensure adequate anticoagulation [12]. The same goes with blood glucose meters. In practice we have observed patients presenting with normal serial glucose readings from their self-monitoring of blood glucose (SMBG) which do not correspond with their clinical presentations or confirmatory checks of glucose values using routine laboratory methods. Some POCT devices come with ready-to-use quality control reagents but these are quickly exhausted or they reach their expiration date. Replacing them is not usually easy although a good number of our respondents claimed they get QC replacement from medical laboratories, instrument vendors and pharmacy shops. Commercially prepared QC materials are usually very expensive to procure, may require reconstitution and a demanding storage condition like temperature requirement. They also have a short shelf life after reconstitution. Owners of POCT devices may not be able to reconstitute and preserve such materials to ensure their viability. It will therefore be easier if the POCT devices are sent to the central laboratory periodically for confirmation of their accuracy especially if the device is used within the hospital.

There is at present no requirement from government governing the procurement, registration and operation of POCT devices in Nigeria. There are two bodies charged with the regulation of operations of Clinical Laboratory testing in Nigeria: The Medical and Dental Council of Nigeria established in 1960 by an act of Parliament (Act Cap 221) now Cap M8 Laws of the Federation of Nigeria 1990, has a mandate to regulate and control laboratory medicine practice [13]. The Medical Laboratory Science Council of Nigeria (MLSCN) which was established by parliament (Act 11 of 2003) is also charged with responsibility to regulate the practice of medical laboratory science in Nigeria [14]. None of these bodies was mandated to regulate the use of POCT devices or extra-laboratory testing. The National Agency for Food and Drug Administration and Control (NAFDAC) is an Agency of the Federal Government charged with the regulation of food, drug and medical devices, etc [15]. It is plausible to assume that included among 'medical devices' are all POCT devices. However, NAFDAC activities have been centred on the regulation of food and drugs with little or no regulation of IVD including POCT devices. Available POCT devices in Nigeria do not carry any indication that they were registered by NAFDAC or any other regulatory agency as seen with other food and drug products. It does appear therefore that Nigerians rely more on the certification of POCT devices by regulatory bodies from countries where these devices were produced. As noted earlier, this may not be reliable due to differences in atmospheric conditions such as heat, humidity, barometric pressure changes, altitude (if applicable), sunlight, surface angle, device movement etc.
Over three-quarter of respondents agreed that there is a need for some form of regulation of use of POCT devices by Government or by Hospital Management (especially if such devices are used within a hospital environment). Within our hospital, there is no committee in place to regulate the use of such devices. For example there is no process to recommend or approve POCT device for use by various departments or individuals managing patients nor is there a recommended process to validate and monitor results generated by such devices. There are no formal training and certification of POCT device operators. There are also issues involving the storage of results generated from such devices for future reference which is presently taken care of by POC connectivity in some hospitals. Other issues to be addressed include: harnessing the revenue generated from such test, pricing of the tests to prevent patient exploitation and a lot more. All these issues can be addressed by inaugurating a POCT Committee in the hospital.

\section{CONCLUSION}

Point of care testing is practiced within and outside our hospitals. The range of analytes that will be available to POCT will continue to increase as technology improves. Although this study shows that majority of operators of POCT devices are medics and paramedics, there is likelihood that many persons with non-medical background are using POCT devices at homes, in registered medical laboratories and even pharmaceutical shops. We recommend that there should be a form of regulation on the use of POCT devices within the hospital by Management. Operators should be trained formally through a training program set up by management. There should be periodic calibration of the instrument by the central laboratory and provision of quality control materials. Government may want to be more proactive by regulating the brands that are allowed to be imported to the country. This can be done by NAFDAC with input from the MDCN and MLSCN. Professional associations including College of Nigerian Pathologists (CNP) should formulate programs to train and certify operators of POCT devices in Nigeria. Efforts should be made to establish and promote POC connectivity - link between results generated by POCT devices with the pool of results generated by the central laboratory to aid easy retrieval for future use. This will go a long way to improve overall patient care and save the cost of an erroneous results.

\section{REFERENCES}

1. Becich, M. J. (2000). Information management: moving from test results to clinical information. Clinical leadership \& management review: the journal of CLMA, 14(6), 296.

2. Point-of-care-testing. (2018). Available at http:/en.wikipedia.org/wiki/. Retrieved $23^{\text {rd }}$ December 2018

3. O’Kelly, R. A., Brady, J. J., Byrne, E., Hooley, K., Mulligan, C., Mulready, K., \& Boran, G. (2011). A 
survey of point of care testing in Irish hospitals: room for improvement. Irish journal of medical science, 180(1), 237-240.

4. Howerton, D., Anderson, N., Bosse, D., Granade, S., Westbrook, G., (2015). Good laboratory practice for waived testing sites: survey findings from testing sites holding a certificate of waiver under the Clinical Laboratory Improvement Amendments of 1988 and recommendations for promoting quality testing. MMWR. 2005; 54(RR13):1-25. Available at www.cdc.gov/mmwr. Retrieved 23rd December 2018.

5. CDC. (2005). Transmission of hepatitis B virus among persons undergoing blood glucose monitoring in long-term-care facilitiesMississippi, North Carolina, and Los Angeles Country, California, 2003-2004. MMWR. 54, 220223.

6. Clinical Laboratory Improvement Amendments of 1988, 42 USC. 263a PL100-578.

7. Laboratory Requirements 42 CFR. (2003) Chapter 4 Part 493

8. U.S. Department of Health and Human Services, Office of Inspector General. Enrolment and certificate processes in the clinical laboratory improvement amendments program. Washington DC US. Department of Health and Human Services. (2001). Available at http://org.oig.hhs.gov/oei/reports/oei-05-0000251.pdf. Retrieved 23rd December 2018.

9. Katayer, A., Belciza, C., Seccombe, D. W. (2010). Establishment of reference interval for clinical laboratory test results: is there a better way?
American Journal of Clinical Pathology, 133(2), 180-186.

10. Food and Drug Administration Guidance Document. (2008). Recommendations for Clinical Laboratory Improvement Amendments of 1988 (CLIA) Waiver Applications for Manufacturers of In Vitro Diagnostic Devices. Jan 30. 2008 Available at http://www.fda.gov/MedicalDevices/DeviceRegula tionandGuidance/GuidanceDocum ents/ucm079632.htm. Retrieved June 13th 2019

11. American Association of Clinical Chemistry. Point-Of-Care Testing Professional Certification. (2018). Available at: https://www.aacc.org/education-and-career/aacccertification/point-of-care-testing-professionalcertification. Retrieved 14th June 2019.

12. van den Brule, J. M., Romkes, J. H., Brouwer, M. A., \& Noyez, L. (2010). An error in the CoaguChek $S \circledR$ device results in prosthetic heart valve thrombosis. Interactive cardiovascular and thoracic surgery, 11(1), 123-124.

13. Medical and Dental Council of Nigeria. MDCN Mandate. Available at https://mden.gov.ng/page/about-us/mden-missionvision. Retrieved $14^{\text {th }}$ June 2019.

14. Medical Laboratory Science Council of Nigeria. MLSCN Mandate. Available at www.mlscn.gov.ng/index.php/mlscn.mandate. Retrieved $14^{\text {th }}$ June 2019.

15. National Agency for Food and Drug Administration and Control. Drug and Medical Devices. Available at https://www.nafdac.gov.ng. Retrieved $14^{\text {th }}$ June 2019. 